\title{
A Multichannel THz Detector Using Integrated Bow-Tie Antennas
}

\author{
Hairui Liu, ${ }^{1,2}$ Junsheng Yu, ${ }^{1}$ Peter Huggard, ${ }^{2}$ and Byron Alderman ${ }^{2}$ \\ ${ }^{1}$ Beijing University of Posts and Telecommunications, Beijing 100876, China \\ ${ }^{2}$ MMT Group, RAL Space, STFC Rutherford Appleton Laboratory, Didcot OX11 0QX, UK
}

Correspondence should be addressed to Hairui Liu; liuhairui1983@126.com

Received 7 November 2012; Accepted 10 April 2013

Academic Editor: Wenhua Chen

Copyright (c) 2013 Hairui Liu et al. This is an open access article distributed under the Creative Commons Attribution License, which permits unrestricted use, distribution, and reproduction in any medium, provided the original work is properly cited.

\begin{abstract}
This paper presents a kind of a multichannel $\mathrm{THz}$ detector using lens-based bow-tie array. A hyperhemispherical silicon lens is employed to provide a focal plane; 8 bow-tie elements are arranged on the focal plane with careful design to show a performance of broadband, high gain, well compact, and easy assembling. These characteristics of the detector are preferred for detecting weak $\mathrm{THz}$ signal. Measured far field shows that the radiation pattern of each element is shifted angularly, by $\approx 9^{\circ}$, which can be used for $\mathrm{THz}$ imaging. Tested responsivity of the detector shows a good spectral performance from 260 to $400 \mathrm{GHz}$ : respective values were $\geq 220 \mathrm{~V} / \mathrm{W}$, and the best NEP is achieved at about $60 \mathrm{pW} / \sqrt{\mathrm{Hz}}$. Besides that, the proposed antenna has advantages of simple structure, easy fabrication, and low cost.
\end{abstract}

\section{Introduction}

Terahertz radiation is frequently treated as the spectral region within frequency range of 1-10 THz. It has shown unique properties and proved a variety of applications, such as concealed weapon detection, spectroscopy, short-range radar, and secured high-speed data communication [1-3]. For these applications, $\mathrm{THz}$ detector is an essential component. Detectors based on Schottky diode can operate at room temperature and have an extremely fast response time compared with other detectors, such as microbolometers [4] or Golay cells [5]. In order to couple the weak $\mathrm{THz}$ radiation to Schottky diodes, high-gain antennas are preferred. Waveguide-based detectors connected with horns can provide high gain with directivity of $22-25 \mathrm{~dB}$; however, the operating frequency is limited by the cut-off frequency of waveguide [6]. A series of detectors have been designed by using self-complementary antennas mounted on silicon lenses, exhibiting a broadband response and achieving a directivity of up to $30 \mathrm{~dB}$ [7-10]. This kind of detector is a good option for $\mathrm{THz}$ imaging with the advantages of low cost, broadband, and fast response. This imaging system is typically done by a single detector with scanning optics [11, 12]; however, mechanical scanning system is always time consuming, costly, and with a large physical size. Detectors by using integrated antennas to provide multichannel can significantly simplify the imaging system and save time. A three-element linear array of doubleslot antennas was fabricated and measured on a silicon lens at $258 \mathrm{GHz}$ [13]. More recently, a $2 * 2$ array of $280 \mathrm{GHz}$ Schottky-barrier diode detectors using an on-chip patch antenna is fabricated in a $130 \mathrm{~nm}$ logic CMOS process and integrated into a 2D focal-plane image scanner [14].

We have done some research on $\mathrm{THz}$ generation and detection [16]. A compact photonic system is used to provide video-rate $\mathrm{THz}$ signal. It has advantages of low cost and high speed; however, it exhibits a low power. In order to detect this weak $\mathrm{THz}$ radiation for imaging, a high-gain and compact integrated antenna is employed to provide multioutput. It utilizes an 8-element linear array of bow-tie antennas mounted on a hyperhemispherical silicon lens. The bow-tie antenna is well considered according to the requirements of the detector. Several self-complementary antennas could provide broadband performance, such as spiral antennas [8] and logperiodic antennas [7]. But for compact integrated antenna, it should be with the advantages of small size, enough area for soldering Schottky diode, and linear polarization. For these reasons, bow-tie antennas are preferred for this $\mathrm{THz}$ detector.

In the following, we will describe the design and measurements of the multichannel $\mathrm{THz}$ detector. In Section 2, diode characteristics and detector circuit are presented to show the 


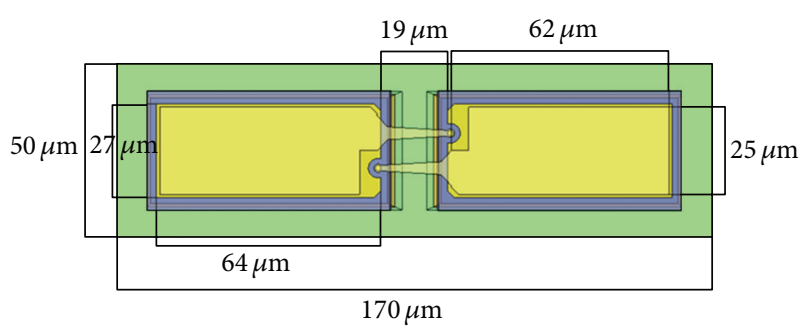

FIGURE 1: Dimensions of the antiparallel Schottky diode chip. The airbridges are approximately $19 \mu \mathrm{m}$ long. The chip is supported on GaAs substrates [15].

principle of rectifying signal. Section 3 shows the antenna design and simulation. In Section 4, measurements including spectral responsivity and far-field pattern are presented. Finally conclusions of the detector and antenna performance are discussed in Section 5.

\section{Diode Characteristics and Detector Circuit}

When a THz signal is applied, the Schottky diode generates DC voltage proportional to the signal power due to its second and other even-order terms associated with nonlinearity. It can be used to directly detect the $\mathrm{THz}$ radiation with proper antennas. The antiparallel Schottky diodes were fabricated at STFC Rutherford Appleton Laboratory [15]. The dimension of an antiparallel pair of air-bridged devices is shown in Figure 1. The unsupported length of each airbridge is $19 \mu \mathrm{m}$. Anodes, $1.0 \mu \mathrm{m}$ in diameter, are located at the end of the bridges. The airbridges are made of gold, with a titanium Schottky metallization contact to the GaAs.

When a small AC signal is applied, the Schottky diode generates DC voltage proportional to the signal power due to its square law $I-V$ performance. The square law $I-V$ relationship is caused by the dynamic resistance $R_{j}$ of the Schottky contact, $R_{j}=d V / d I$. The series resistance $R_{s}$, saturation current $I_{0}$, and ideality factor $\eta$ are determined by data fitting with the classical Schottky model [19]:

$$
I(V)=I_{0}\left(\exp \left(\frac{q\left(V-R_{s} I\right)}{\eta K_{B} T}\right)-1\right)
$$

where $q$ is the electron charge, $T=293.1 \mathrm{~K}$, and $K_{B}$ is Boltzmann's constant $1.38 \cdot 10-23 \mathrm{~J} / \mathrm{K}$. Respective values are $R_{s}=$ $13.67 \Omega, I_{0}=0.98 \mathrm{fA}$, and $\eta=1.18$. For our diode model, the zero voltage junction capacitance $C_{j}$ is around $1.42 \mathrm{fF}$ related to the anode area of $0.95 \mu \mathrm{m}^{2}$, which results in a high cut-off frequency, $f_{T}=1 / 2 \pi R_{s} C_{j} \approx 8 \mathrm{THz}$. The intrinsic voltage responsivity of the diode $R_{v}$ can be determined from the diode $I-V$ equation [19],

$$
R_{v}=\frac{d^{2} V / d I^{2}}{2(d V / d I)}=\frac{1}{2\left(I_{d}+I_{0}\right)} .
$$

From (2), the voltage responsivity peaks when bias current is close to zero. But this is not desired because low reverse saturation current brings a high junction resistance $(>1 \mathrm{M} \Omega)$,

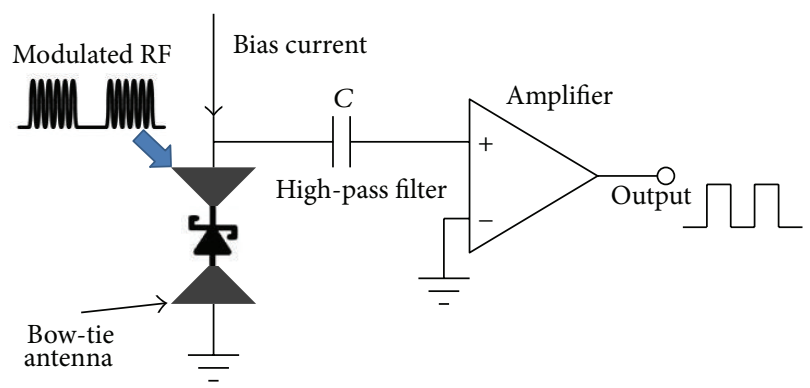

FIGURE 2: Simplified detector circuit.

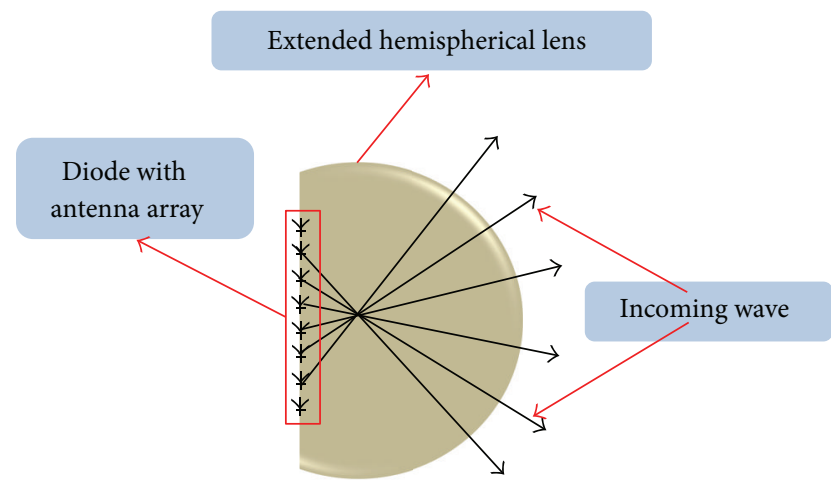

FIGURE 3: Schematic diagram of the compound antenna.

which is too high for efficient power matching to the diode [14]. In this case, the diode in the detector design is forward biased. The simplified detector circuit is shown in Figure 2. The bias current is around $150 \mu \mathrm{A}$ which keeps the $R_{j}$ closed to $200 \Omega$. The modulated $\mathrm{THz}$ signal illuminates the bow-tie antenna and couples to the diode. Then, the rectified signal passes the filter and goes to the low-noise amplifier.

\section{Antenna Design and Simulation}

The antenna used for coupling the THz radiation to Schottky diodes is shown in Figure 3. Incoming wave is focused by a high dielectric constant silicon lens into the focal plane. By linearly arranging the detector diodes with a proper antenna, a large-scan angle is obtained for imaging.

The antenna array used on the focal plane is supported by a $0.15 \mathrm{~mm}$ thick quartz substrate. Bow-tie element is chosen to provide a broadband response, symmetrical far-field pattern, linear polarization, and large area for diode soldering. The feed gap is $0.03 \mathrm{~mm} * 0.03 \mathrm{~mm}$ for soldering Schottky diode. In order to resonate on $300 \mathrm{GHz}$, the arm length should be close to $1 / 4$ wavelength of the medium, taking into account the quartz dielectric constant: 3.78 . The flare angle is optimized to achieve a symmetric far-field pattern. By using Ansoft High-Frequency Structure Simulator (HFSS), the whole size of the bow-tie element is $0.21 \mathrm{~mm} * 0.1 \mathrm{~mm}$ as shown in Figure 4.

The geometry of bow-tie element array is displayed in Figure 5. Eight elements are arranged in parallel with bowtie arms; this arrangement provides a lower mutual coupling 


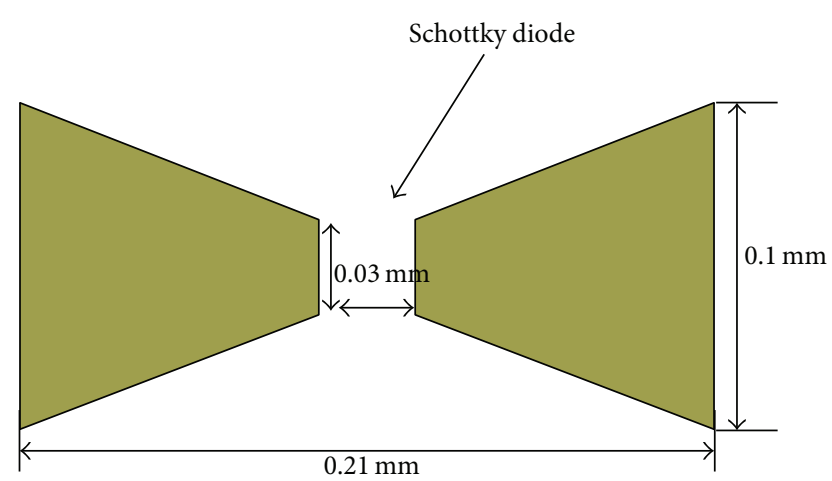

Figure 4: Geometry of the bow-tie element.

between adjacent elements. For an imaging array, each channel of the array should be closely packed to make full use of the focal-plane area. But as the interactions between elements should also be considered, we optimized the spacing of the array using HFSS to achieve a coupling of $-20 \mathrm{~dB}$ or less. This gave an antenna centre-centre separation of $340 \mu \mathrm{m}$. The eight bow-tie antennas and associated conductors are photolithographically patterned in an Au layer deposited on the quartz substrate, as shown in Figure 6. One side of each antenna is connected to a common ground line, which in turn connects to the outmost two pads of the connector array. The other side of the antenna is connected to one of the middle eight pads. The conductors close to the antennas are orthogonal to the E-field to which the antenna is sensitive: this is done to minimise the effect on the radiation pattern. The connectors together with bow-tie elements are simulated using HFSS to calculate the impedance of the bow-tie element. By using diode parameters, $R_{s}, R_{j}$, and $C_{j}$, the matching between diode and antenna can be derived. As shown in Figure 7, considering the spectral impedance of the diode, the $S_{11}$ curve presents undulation growth with frequency. At $300 \mathrm{GHz}$ it is below $-10 \mathrm{~dB}$, and the peak value in about $-4 \mathrm{~dB}$ from $200 \mathrm{GHz}$ to $400 \mathrm{GHz}$.

The coupling between the adjacent elements of the array is simulated using HFSS. As shown in Figure 8, the simulated $S_{21}$ parameter is below $-20 \mathrm{~dB}$ from $260 \mathrm{GHz}$ to $400 \mathrm{GHz}$. Usually the envelope correlation coefficient is an important parameter to evaluate the diversity characteristic of a multiantenna system [20]. The envelope correlation coefficient computed from $S$ parameters is shown as follows [21]:

$$
\rho_{e}=\frac{\left|S_{11}^{*} S_{12}^{*}+S_{21}^{*} S_{22}^{*}\right|^{2}}{\left[1-\left(\left|S_{11}\right|^{2}+\left|S_{21}\right|^{2}\right)\right] 1-\left(\left|S_{22}\right|^{2}+\left|S_{12}\right|^{2}\right)} .
$$

From the simulated $S$ parameters, the envelope correlation coefficient was calculated, as shown in Figure 9. The envelope correlation coefficient has a peak value at $202 \mathrm{GHz}, \leq 0.3$, and typically lower than 0.05 from $210 \mathrm{GHz}$ to $400 \mathrm{GHz}$.

The hyperhemispherical lens is a hemispherical lens with an attached extension length $L, n$ is the index of refraction of the lens, and $R$ is the radius of the lens shown in Figure 10. The hyperhemispherical lens was borrowed into the millimetre wave field from optics [22], and it was found that it could

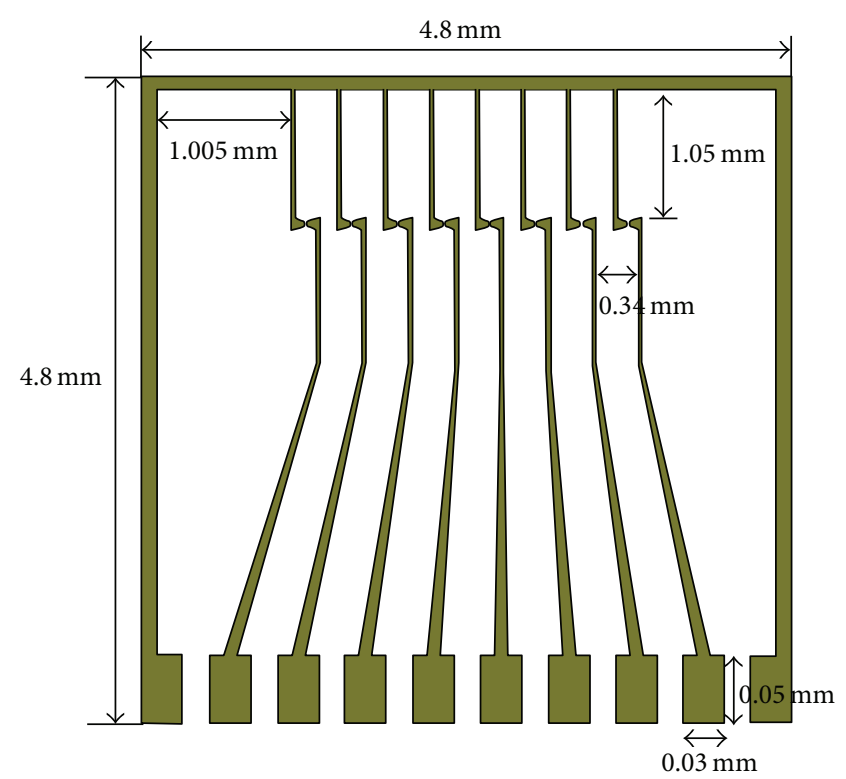

FIGURE 5: Geometry of the bow-tie element array.

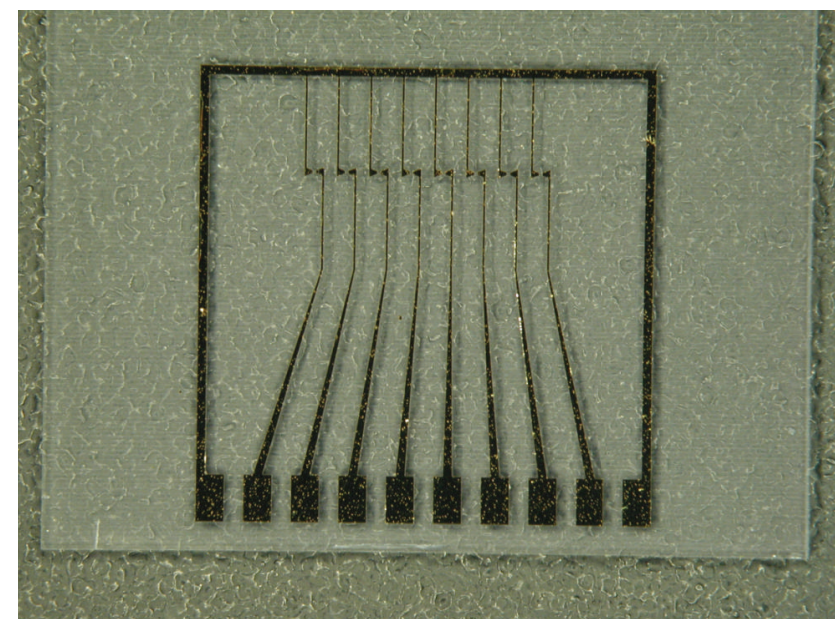

FIGURE 6: Photograph of the bow-tie array on quartz substrate.

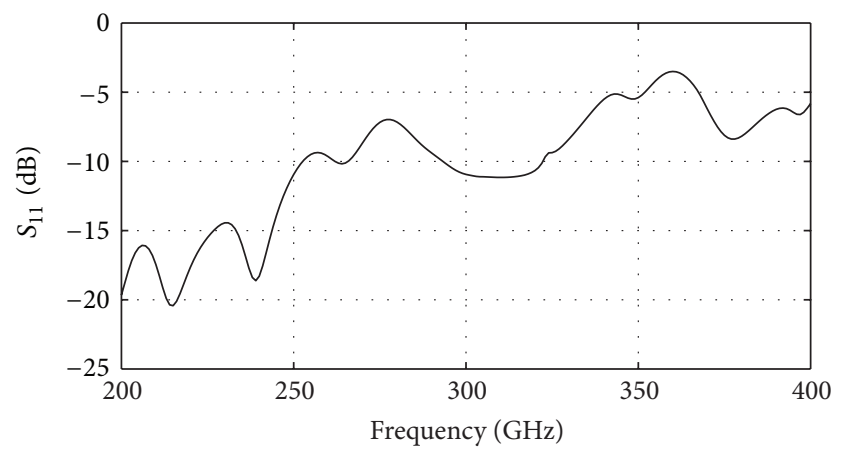

Figure 7: Calculated $S_{11}$ from diode impedance and the antenna impedance. 


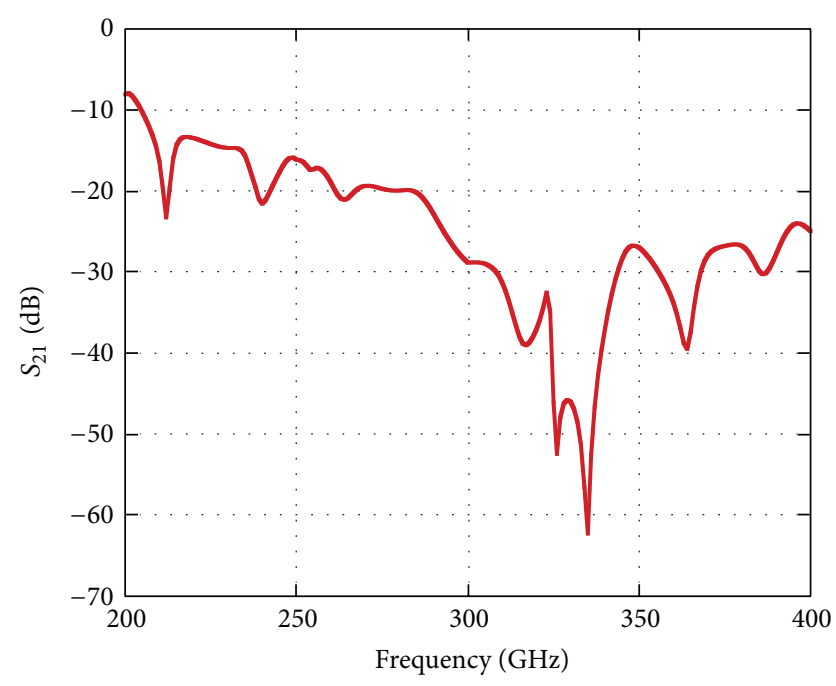

FIgURE 8: Coupling between the adjacent elements.

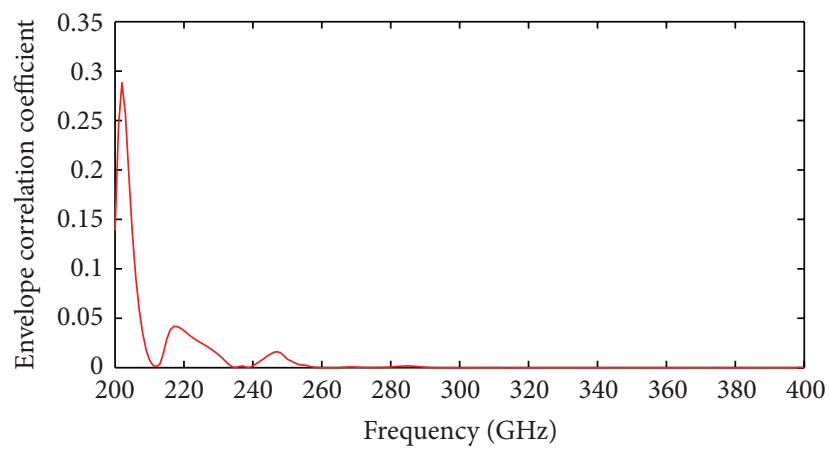

FIGURE 9: Envelope correlation coefficient.

effectively increase the gain of the integrated antenna by $n^{2}$. The hyperhemispherical lens used here has a proper focal area for bow-tie element array, with a diameter of $12 \mathrm{~mm}$, thickness of $7.15 \mathrm{~mm}$, and dielectric constant of 11.7. By taking into account the thickness of the quartz substrate, the extension length is $1.3 \mathrm{~mm}$ with a radius of $6 \mathrm{~mm}$ and gives an $L / R$ of about 0.22 . The comparison of far-field pattern between bowtie element and embedding silicon lens is shown in Figure 11. By using hyperhemispherical, the directivity increased about $10 \mathrm{~dB}$, close to $n^{2}=11.7$. This is done by using ray tracing to illuminate the lens surface with the radiation of bowtie element (computed via HFSS), and Fresnel transmission coefficients are employed to compute the fields across the boundary between lens and the air.

For assembling the antenna, the silicon hyperhemispherical substrate lens is supported by an aluminium bracket and is clamped by a polyethylene cap, as shown in Figure 12. The quartz wafer bearing the antenna array is glued in a machined rectangular depression on the bracket. A ten-pin connector is soldered to the pads on the quartz, which in turn connects to a ribbon cable. The other end of the cable is connected to an eight-channel low-noise preamplifier. Only four channels were populated with Schottky diodes and tested.

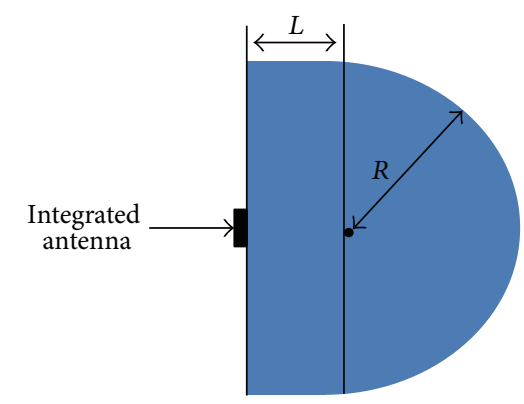

FIGURE 10: Schematic diagram of hyperhemispherical lens.

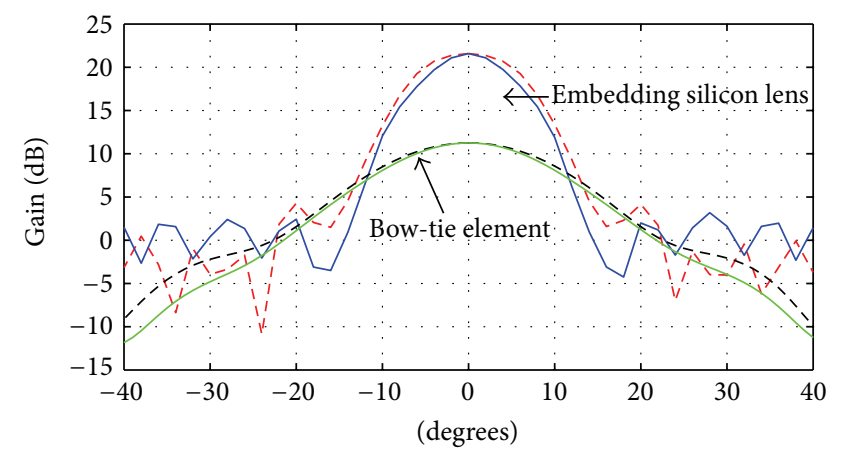

FIGURE 11: Simulated far field of E_plane and H_plane on bow-tie element and embedding silicon lens E_plane (line), H_plane (dashed line).

\section{Measurements and Results}

After antenna assembling, the antenna is soldered with Schottky diodes and connected to amplify circuits. Direct measurement on antenna as a passive component, such as $S$ parameters, is impossible. But the spectral response of the detector can mirror the performance of the antenna. One of the most important performances of detectors is voltage responsivity $R_{v}$, which is mainly decided by the intrinsic responsivity of the diode and the antenna performance.

The responsivity of the detectors was measured on a quasioptical system, as shown in Figure 13. A corrugated horn with a similar aperture with the silicon lens, fed by a frequency-multiplied source [23], delivered a beam into a reflective quasioptical system. The detector was placed at the output beam waist. In this arrangement, almost all the power from the source illuminated the array lens. A terahertz power meter was used to calibrate the output power of the source, which was kept around $20 \mu \mathrm{W}$. The video-rate modulation is done by using an optical chopper with space-duty-cycle ratio of $1: 1$ [24]. This chopper frequency is then used as reference frequency of the lock-in amplifier. The lock-in amplifier measures the rms voltage value of the output of the detector. The expression of the detector voltage responsivity is

$$
R_{v}=\frac{v_{\mathrm{out}}}{P_{\mathrm{in}}}=\frac{(\pi / \sqrt{2}) V_{\mathrm{rms}}}{P_{\mathrm{in}}},
$$




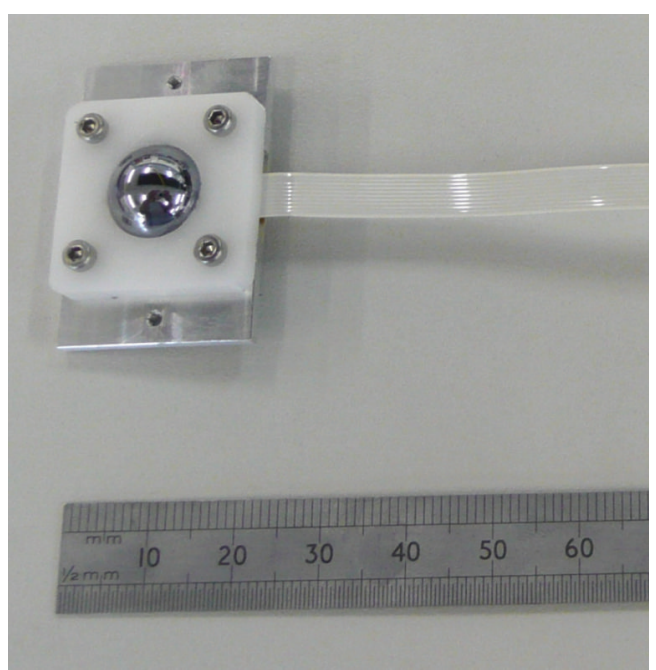

(a) Top view

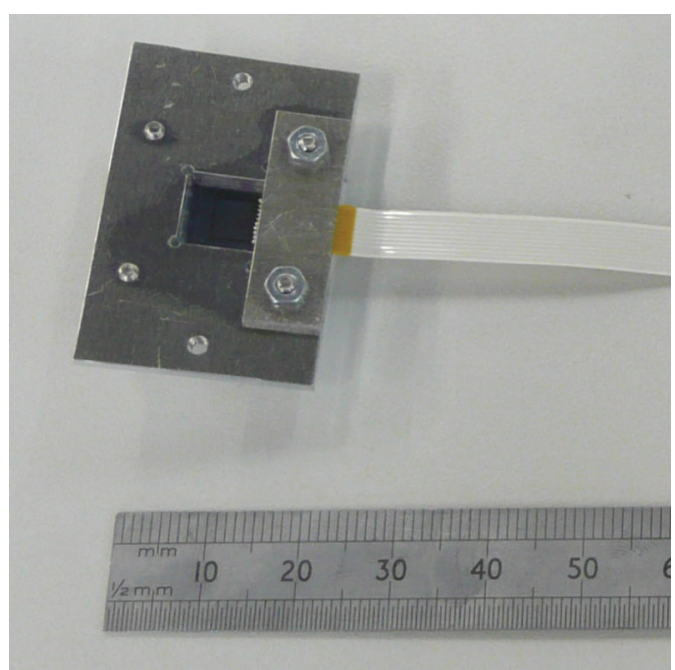

(b) Bottom view

FIgURE 12: Photographs of the fabricated antenna.

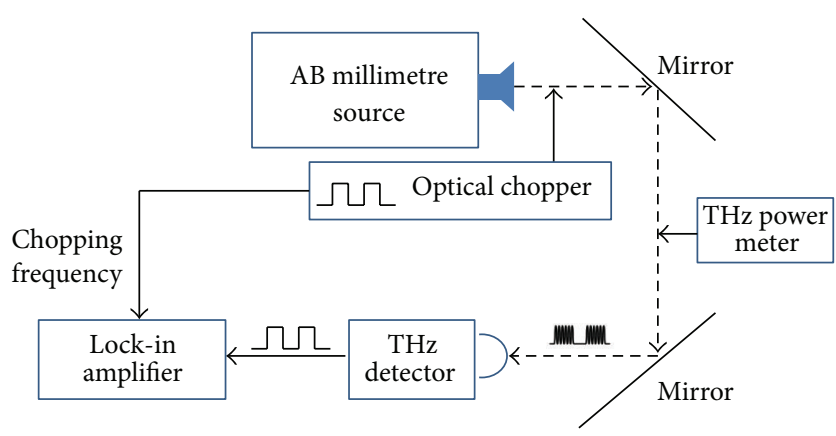

FIGURE 13: Schematic diagram of responsivity measurement setup.

where $P_{\text {in }}$ denotes the input power of the detector measured by terahertz power meter; $v_{\text {out }}$ is $\pi / \sqrt{2}$ times as rms value $V_{\text {rms }}$ measured by lock-in amplifier [25].

From (4), the estimated voltage responsivity versus frequency is calculated and plotted in Figure 14. The responsivity is over $220 \mathrm{~V} / \mathrm{W}$ on the frequency range of $260-400 \mathrm{GHz}$ without preamplifier's gain. The peak responsivity of $330 \mathrm{~V} / \mathrm{W}$ is measured at $300 \mathrm{GHz}$ and meets the predictions of the diode matching. Taking into account 30\% reflection losses, $20 \%$ absorbing losses at the silicon lens [10, 26], antenna coupling mismatch, and nonperfect impedance matching of the diode to the antenna, we arrive at an intrinsic diode responsivity of larger than $2000 \mathrm{~V} / \mathrm{W}$. This is comparable with theoretical estimation.

Another key performance metric for the detector, noiseequivalent power (NEP), is defined as the ratio between the output noise voltage spectral density $(\mathrm{V} / \sqrt{\mathrm{Hz}})$ and the detector voltage responsivity $(\mathrm{V} / \mathrm{W})$. In order to characterise the noise properties, measurements were made on a detector which has the same configuration of the detector array but with a single bow-tie antenna on the quartz. A low-noise opamp amplifier, with typical input noise level of $3.2 \mathrm{nV} / \sqrt{\mathrm{Hz}}$,

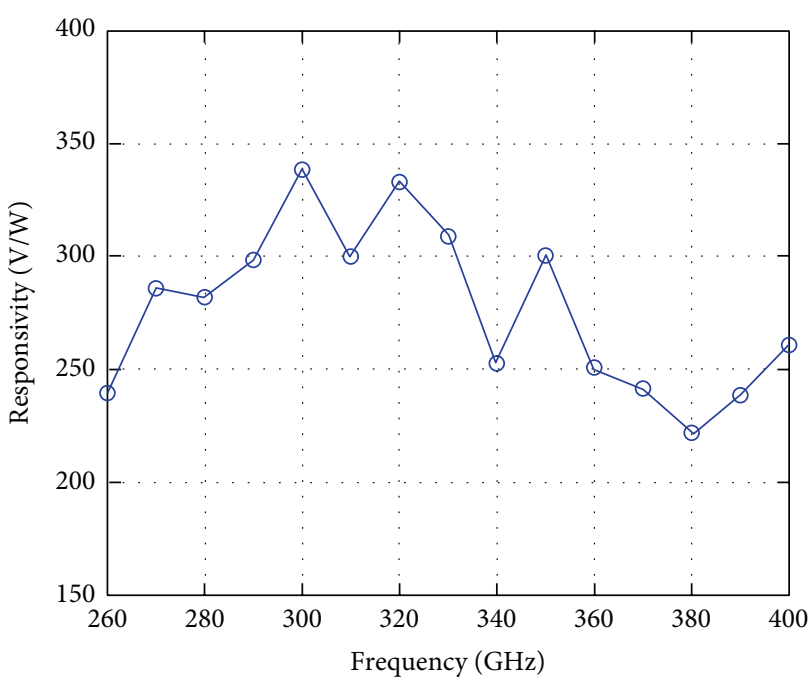

FIGURE 14: Voltage responsivity for one channel of detector.

was used to amplify the signal. The output of amplifier was connected to a lock-in amplifier and spectrum analyser to measure the noise density over different frequency ranges. For the frequency range from $10 \mathrm{kHz}$ to $1 \mathrm{MHz}$, which is the normal video detection modulation rate, the noise is around $20 \mathrm{nV} / \sqrt{\mathrm{Hz}}$. The corresponding NEP is below $100 \mathrm{pW} / \sqrt{\mathrm{Hz}}$ from 260 to $400 \mathrm{GHz}$, and the best NEP is achieved at about $60 \mathrm{pW} / \sqrt{\mathrm{Hz}}$.

The performance of the detector is compared to other similar works using different antennas shown in Table 1. Because of the differences in the tuned frequency, it is difficult to make a clean comparison. Measurements of responsivity show that the proposed detector has a peak value over $330 \mathrm{~V} / \mathrm{W}$ at $300 \mathrm{GHz}, 220-330 \mathrm{~V} / \mathrm{W}$ on the frequency range of 260-400 GHz. It is close to the reports of quasioptical detectors by other groups, using logarithmic spiral antenna [9] 
TABLE 1: Performance of detector compared with prior works.

\begin{tabular}{|c|c|c|c|c|c|}
\hline & Technology & Best NEP $(\mathrm{pW} / \sqrt{\mathrm{Hz}})$ & RES (V/W) & Freq. (THz) & References \\
\hline RAL SBD & QO based on bow-tie & 60 & $220-330$ & $0.26-0.4$ & This work \\
\hline ACST ZBD & QO based on logarithmic spiral & 500 & 200 & $0.05-1$ & {$[9]$} \\
\hline \multirow{2}{*}{ VDI ZBD } & QO based on folded dipole & 20 & $15-280$ & $0.14-0.24$ & [17] \\
\hline & QO based on sinuous & 5 & $300-1000$ & $0.15-0.4$ & {$[18]$} \\
\hline SBD & $130 \mathrm{~nm}$ logic CMOS & 33 & $50-250$ & $0.27-0.29$ & {$[14]$} \\
\hline
\end{tabular}

QO denotes quasioptical detectors; SBD denotes Schottky-barrier diode; ZBD denotes zero bias Schottky diode.

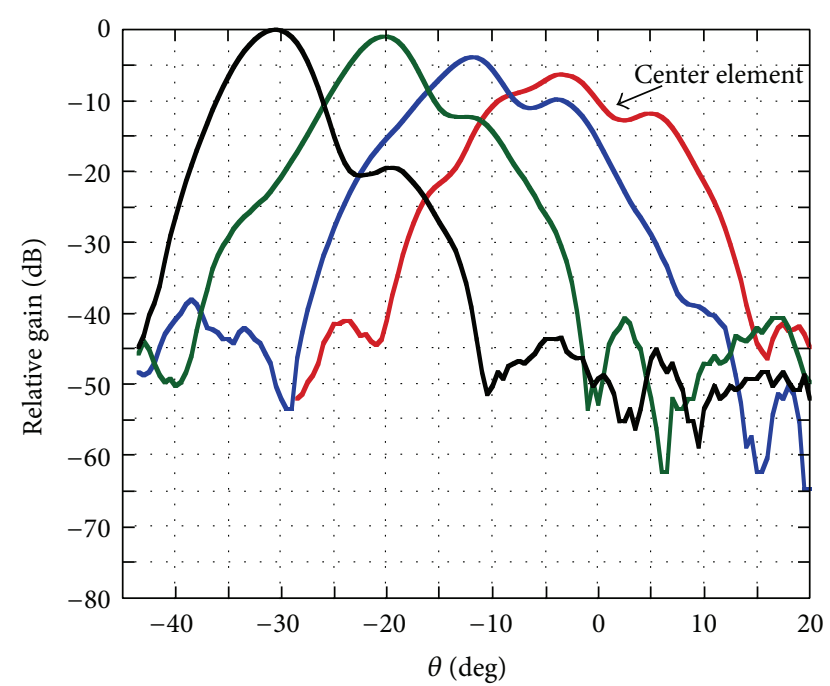

FIGURE 15: Far-field pattern of detector $275 \mathrm{GHz}$.

sinuous antenna [18], and patch antenna [14]. The best NEP of the proposed detector can be achieved at $60 \mathrm{pW} / \sqrt{\mathrm{Hz}}$. This NEP is better than the ACST zero bias detector [9] but worse than the folded dipole detector [17] and $130 \mathrm{~nm}$ logic CMOS detector [14] at their resonant frequency. These performances including responsivity and NEP are highly dependent on the bias current and antenna coupling efficiency. More work will be focused on understanding how they contribute to the responsivity and NEP.

In order to evaluate the imaging performance of the antenna array, far-field pattern of E_plane was measured. This is done by illuminating the detector array in the far field of a broadband frequency multiplier-based source. The detector was fixed on an automated rotation mount. The theta angular $\left(\mathrm{phi}=0^{\circ}\right.$ ) response of each channel was measured separately and results are plotted together. Measurements were made on 4 elements in the same side, and the other 4 elements should have a symmetric pattern. In order to cover a wide frequency range, we tested the pattern of $275 \mathrm{GHz}, 300 \mathrm{GHz}, 330 \mathrm{GHz}$, $350 \mathrm{GHz}$, and $380 \mathrm{GHz}$, which covered more than $100 \mathrm{GHz}$ bandwidth. Since the antenna is combined with detectors, only relative gain is obtained, as shown from Figures 15, 16, 17,18 , and 19. For each element of the detector, the radiation pattern is shifted angularly, by $\approx 9^{\circ}$ with $0.34 \mathrm{~mm}$ spacing on focal plane. This shifted angle is almost linear with displacement off-axis, as shown in Figure 20, which exhibits excellent

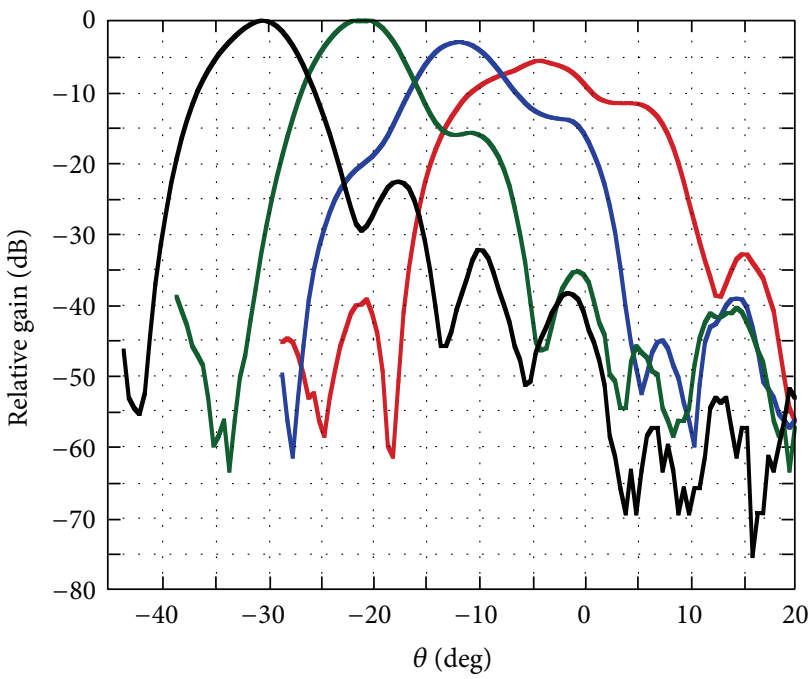

FIGURE 16: Far-field pattern of detector $300 \mathrm{GHz}$.

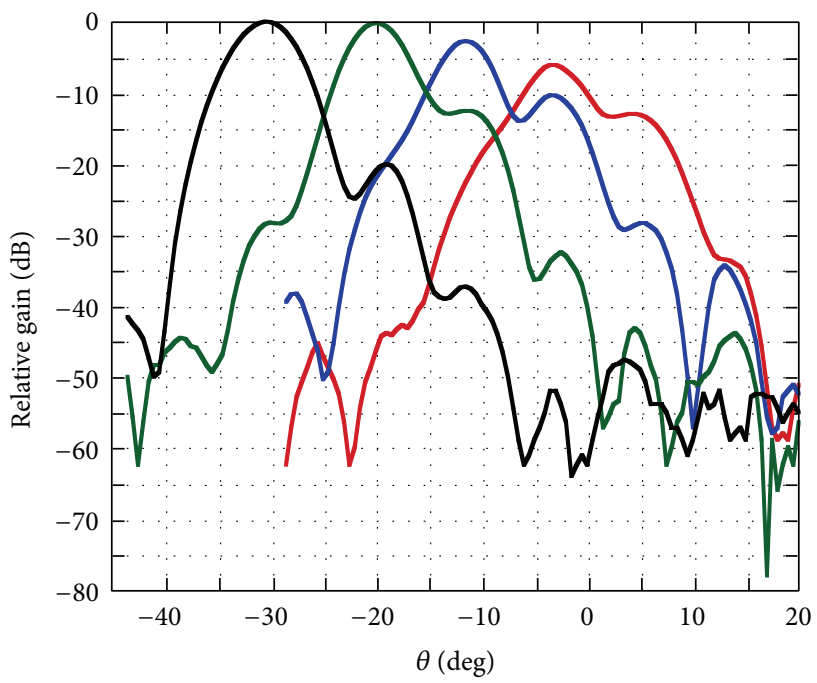

FIGURE 17: Far-field pattern of detector $330 \mathrm{GHz}$.

performance for $\mathrm{THz}$ imaging. At each frequency, it is found that there is an inhomogeneity of pattern between different elements, such as maximum gain and beam width. This is mainly due to two reasons. The first is the slight difference in the diode characteristics affecting the responsivity of the element. The other is that the pattern changes with the 


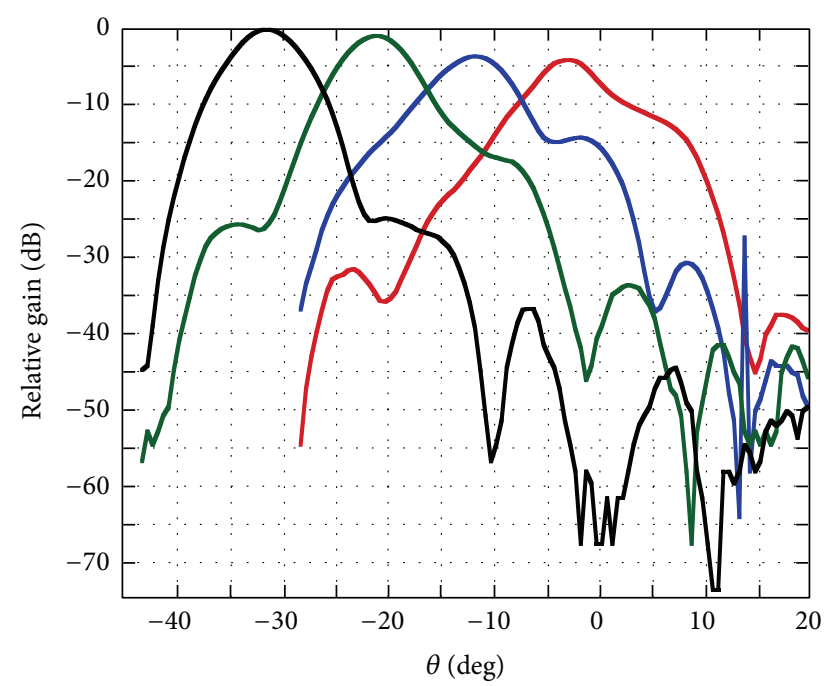

FIGURE 18: Far-field pattern of detector $350 \mathrm{GHz}$.

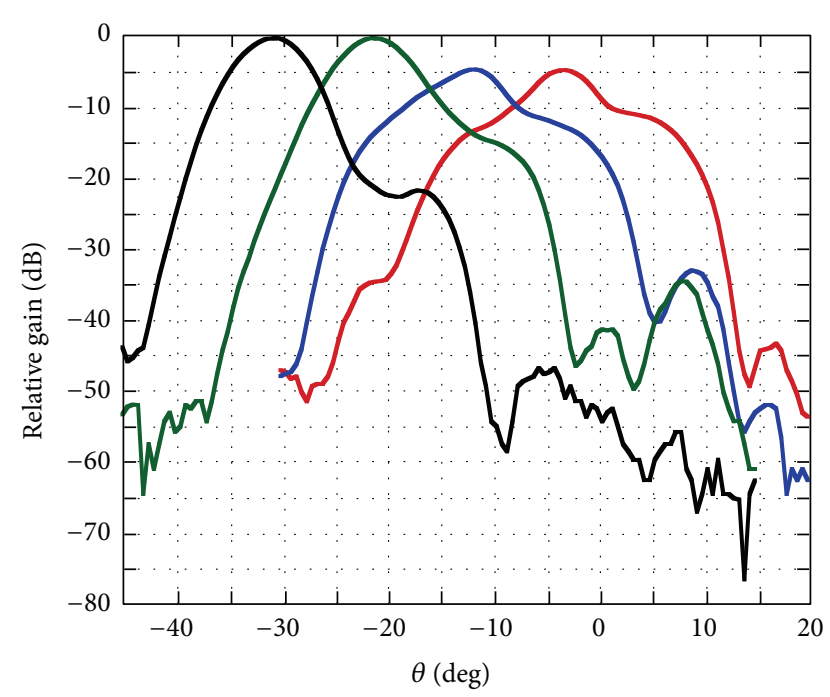

FIGURE 19: Far-field pattern of detector $380 \mathrm{GHz}$.

displacement on focal plane [27]. This can be explained by the function between directivity and the ratio of $L / R$. The corresponding peak directivity as a function of $L / R$ is shown in Figure 21; the maximum directivity is achieved when $L / R$ is near 0.34 . This calculation meets the report of [10], which used a double-slot antenna on hyperhemispherical silicon lens. When the feed antenna is in centre of the focal plane, the $L / R$ is 0.22 for the proposed antenna and provides a directivity of $21 \mathrm{~dB}$. When moving the feed antenna position off-axis, the equivalent $L$ (distance between feed antenna and the centre of hemispherical lens) is longer. Therefore when $L / R$ increases with displacement off-axis, the antenna array exhibits a higher gain on the outmost element. In order to reduce the differences in gain and shape between each element, the ratio of $L / R$ should be optimized to 0.34 . In this case when moving the feed antenna position off-axis, the antenna gain changes slightly with increasing $L / R$.

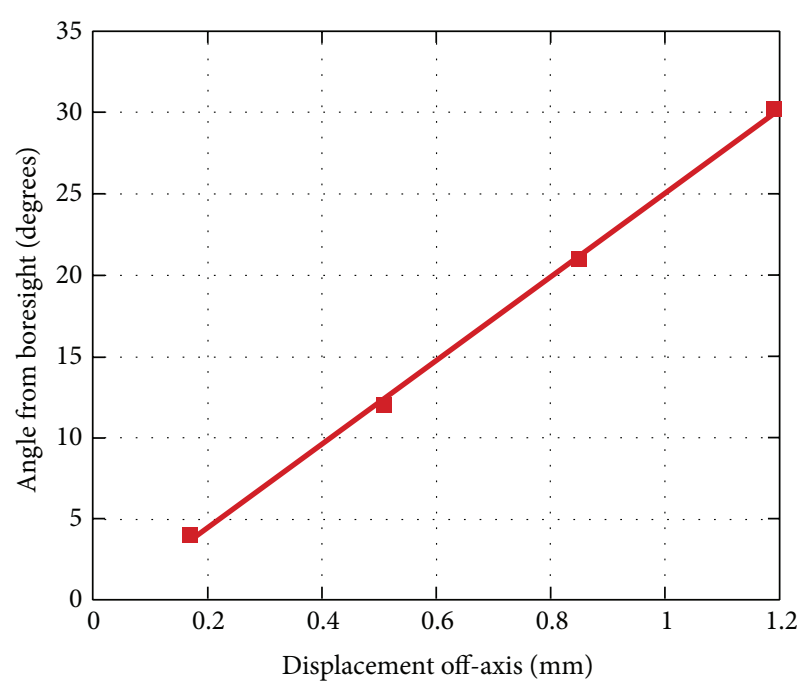

FIGURE 20: Shifted angle versus off-axis displacement.

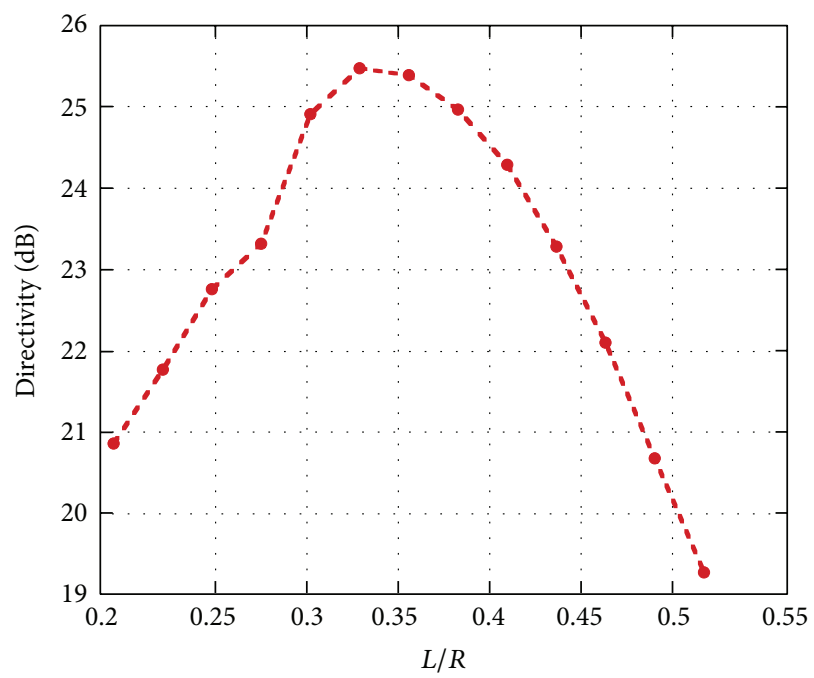

FIGURE 21: Directivity of hyperhemispherical lens as a function of $L / R$ at $300 \mathrm{GHz}$.

\section{Conclusions}

In the paper, a multichannel $\mathrm{THz}$ detector using integrated bow-tie antennas is presented. It employed hyperhemispherical silicon lens to provide a high gain more than $20 \mathrm{~dB}$ and bow-tie element array to provide broadband performance. Simulation of $S$ parameters is made to show that the isolation characteristic $\left(S_{21}\right)$ is less than $-20 \mathrm{~dB}$ between adjacent elements from $260 \mathrm{GHz}$ to $400 \mathrm{GHz}$. This brings an envelope correlation coefficient of this antenna far less than 0.5 which leads to a good diversity characteristic for MIMO application. Measurements of responsivity and far field were made on the $\mathrm{THz}$ detector. Results of responsivity show that the detector has a good spectral response from $260 \mathrm{GHz}$ to $400 \mathrm{GHz}$ : respective values were $\geq 220 \mathrm{~V} / \mathrm{W}$, and the best NEP is achieved at about $60 \mathrm{pW} / \sqrt{\mathrm{Hz}}$. Far-field pattern shows that the radiation of each element is shifted angularly, by $\approx 9^{\circ}$ with 
$0.34 \mathrm{~mm}$ spacing, which exhibits excellent performance for $\mathrm{THz}$ imaging. The antenna structure is highly compact with low cost and easy assembling, which is promising for $\mathrm{THz}$ imaging.

\section{Acknowledgments}

This work is funded by the UK's STFC Centre for Instrumentation. The authors are very grateful to Hui Wang, Matthew Oldfield, and Hosh Sanghera, all at STFC's Rutherford Appleton Laboratory (RAL), for their immense contributions to this project.

\section{References}

[1] P. H. Siegel, "Terahertz technology", IEEE Transactions on Microwave Theory and Techniques, vol. 50, no. 3, pp. 910-928, 2002.

[2] S. Yeom, D. S. Lee, J. Y. Son et al., "Distance estimation of concealed objects with stereoscopic passive millimeter-wave imaging," Progress in Electromagnetics Research, vol. 115, pp. 399-407, 2011.

[3] S. Oka, H. Togo, N. Kukutsu, and T. Nagatsuma, "Latest trends in millimeter-wave imaging technology," Progress in Electromagnetics Research Letters, vol. 1, pp. 197-204, 2008.

[4] F. J. González, M. A. Gritz, C. Fumeaux, and G. D. Boreman, "Two dimensional array of antenna-coupled microbolometers," International Journal of Infrared and Millimeter Waves, vol. 23, no. 5, pp. 785-797, 2002.

[5] Goley cell, Tydex, http://www.tydex.ru/products/thz_optics/golay_cell/.

[6] J. L. Hesler and T. W. Crowe, "NEP and responsivity of THz zero-bias Schottky diode detectors," in Proceedings of the 32nd International Conference on Infrared and Millimetre Waves, and 15th International Conference on Terahertz Electronics (IRMMW-THz '07), pp. 844-845, September 2007.

[7] J. L. Hesler, L. Liu, H. Xu, Y. Duan, and R. M. Weikle, “The development of Quasi-optical THz detectors," in Proceedings of the 33rd International Conference on Infrared, Millimeter and Terahertz Waves (IRMMW-THz '08), pp. 1-2, September 2008.

[8] D. Schoenherr, C. Bleasdale, T. Goebel et al., "Extremely broadband characterization of a Schottky diode based THz detector," in Proceedings of the 35th International Conference on Infrared, Millimeter, and Terahertz Waves (IRMMW-THz '10), pp. 1-2, September 2010.

[9] A. Semenov, O. Cojocari, H. W. Hübers, F. Song, A. Klushin, and A. S. Müller, "Application of zero-bias Quasi-optical Schottkydiode detectors for monitoring short-pulse and weak terahertz radiation," IEEE Electron Device Letters, vol. 31, no. 7, pp. 674676, 2010

[10] D. F. Filipovic, S. S. Gearhart, and G. M. Rebeiz, "Doubleslot antennas on extended hemispherical and elliptical silicon dielectric lenses," IEEE Transactions on Microwave Theory and Techniques, vol. 41, no. 10, pp. 1738-1749, 1993.

[11] S. Barbieri, J. Alton, C. Baker, T. Lo, H. E. Beere, and D. Ritchie, "Imaging with $\mathrm{THz}$ quantum cascade lasers using a Schottky diode mixer," Optics Express, vol. 13, no. 17, pp. 6497-6503, 2005.

[12] I. Kasalynas, D. Seliuta, R. Simniskis, V. Tamosiunas, K. Kohler, and G. Valusis, "Terahertz imaging with bow-tie InGaAs-based diode with broken symmetry," Electronics Letters, vol. 45, no. 16, pp. 833-835, 2009.
[13] S. S. Gearhart and G. M. Rebeiz, "A monolithic $250 \mathrm{GHz}$ Shottky-diode receiver," IEEE Transactions on Microwave Theory and Techniques, vol. 42, no. 12, pp. 2504-2511, 1994.

[14] R. Han, Y. Zhang, D. Coquillat et al., "A 280-GHz Schottky diode detector in 130-nm digital CMOS," IEEE Journal of Solid-State Circuits, vol. 46, no. 11, pp. 2602-2612, 2011.

[15] http://www.teratechcomponents.com/.

[16] H. Liu, J. Yu, P. G. Huggard, and B. Alderman, "Measurements of Schottky-diode based THz video detectors," Terahertz Science and Technologh, vol. 4, no. 3, pp. 104-108, 2011.

[17] L. Liu, H. Xu, Y. Duan, A. W. Lichtenberger, J. L. Hesler, and R. M. Weikle, "A $200 \mathrm{GHz}$ Schottky diode Quasi-optical detector based on folded dipole antenna," in Proceedings of the 20th International Symposium on Space Terahertz Technology, Charlottesville, Va, USA, April 2009.

[18] L. Liu, J. L. Hesler, H. Xu, A. W. Lichtenberger, and R. M. Weikle, "A broadband Quasi-optical terahertz detector utilizing a zero bias Schottky diode," IEEE Microwave and Wireless Components Letters, vol. 20, no. 9, pp. 504-506, 2010.

[19] A. M. Cowley and H. O. Sorensen, "Quantitative comparison of solid-state microwave detectors," IEEE Transactions on Microwave Theory and Techniques, vol. 14, no. 12, pp. 588-602, 1966.

[20] S. C. K. Ko and R. D. Murch, "Compact integrated diversity antenna for wireless communications," IEEE Transactions on Antennas and Propagation, vol. 49, no. 6, pp. 954-960, 2001.

[21] S. Blanch, J. Romeu, and I. Corbella, "Exact representation of antenna system diversity performance from input parameter description," Electronics Letters, vol. 39, no. 9, pp. 705-707, 2003.

[22] D. B. Rutledge and M. S. Muha, "Imaging antenna arrays," IEEE Transactions on Antennas and Propagation, vol. 30, no. 4, pp. 535-540, 1982.

[23] http://www.abmillimetre.com/index.htm.

[24] http://www.edmundoptics.com/electro-optics/electro-opticsaccessories/optical-chopper/2223.

[25] "Understanding measurements using an oscilloscope versus a lock-in amplifier," Spectrum Detector Inc., Lake Oswego, Ore, USA, Application Note 1009, http://www.spectrumdetector.com.

[26] M. V. Afsar and K. J. Button, "Millimeter wave dielectric properties of materials," in Infrared and Millimeter Waves, vol. 12, chapter 1, Academic Press, New York, NY, USA, 1984.

[27] D. F. Filipovic, G. V. Eleftheriades, and G. M. Rebeiz, "Off-axis imaging properties of substrate lens antennas," in Proceedings of the 5th International Symposium on Space Terahertz Technology, pp. 778-787, 1994. 

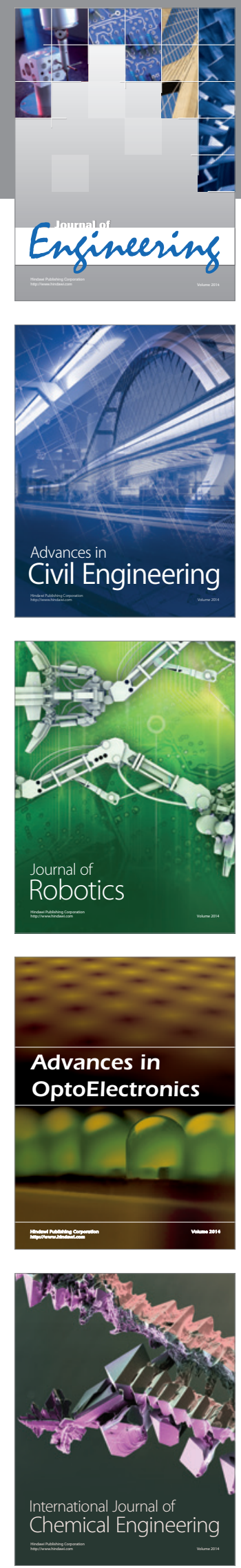

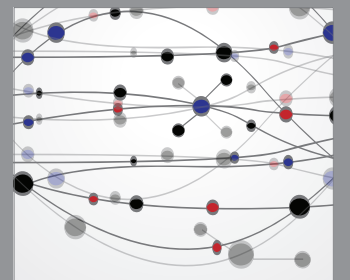

The Scientific World Journal
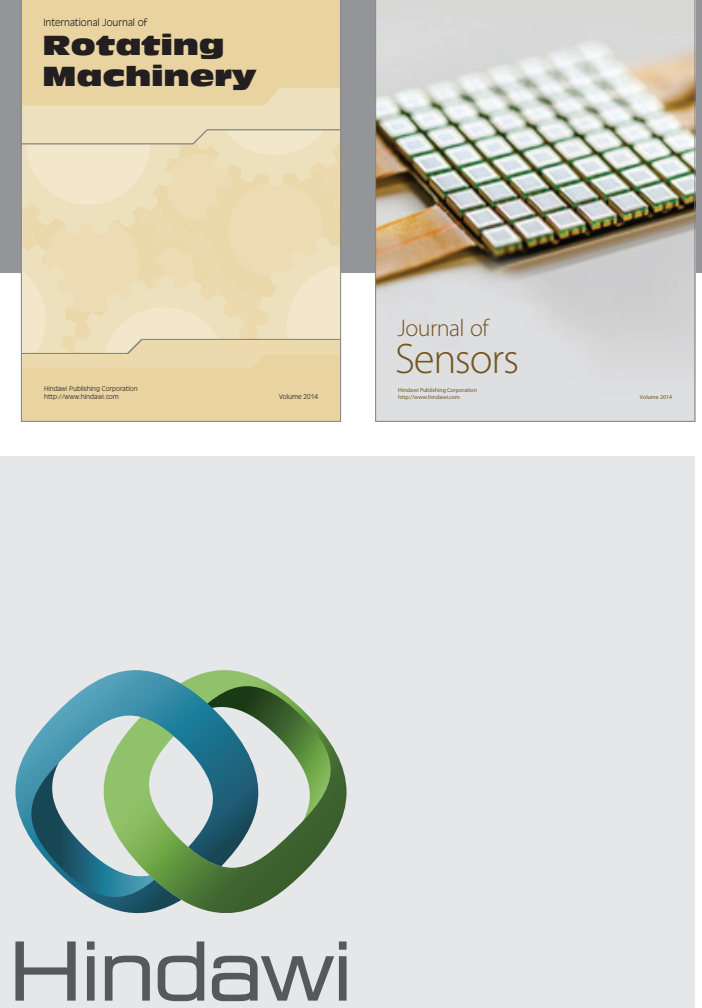

Submit your manuscripts at http://www.hindawi.com
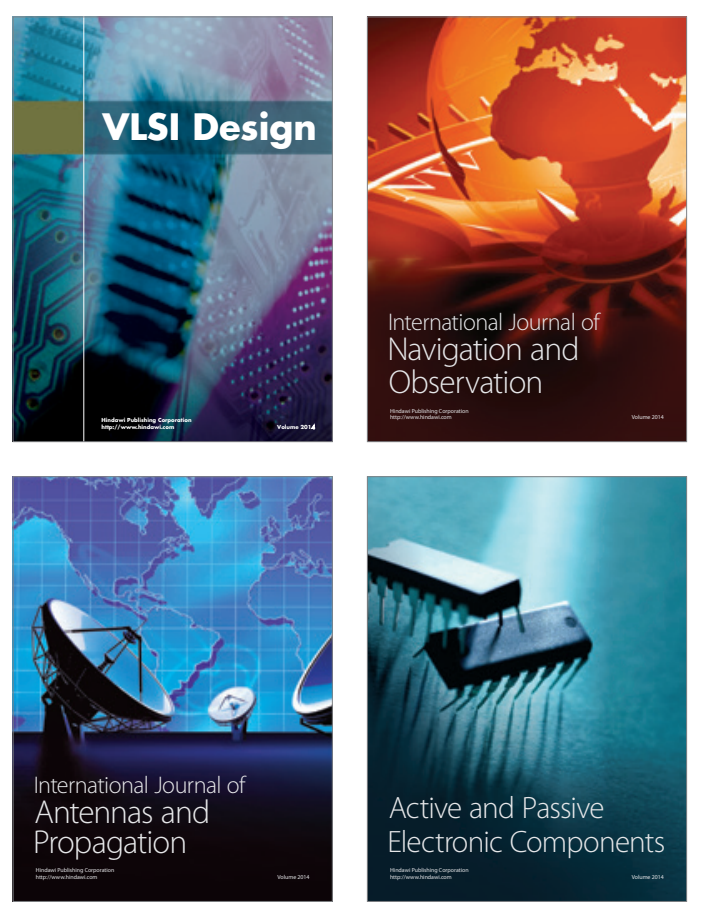
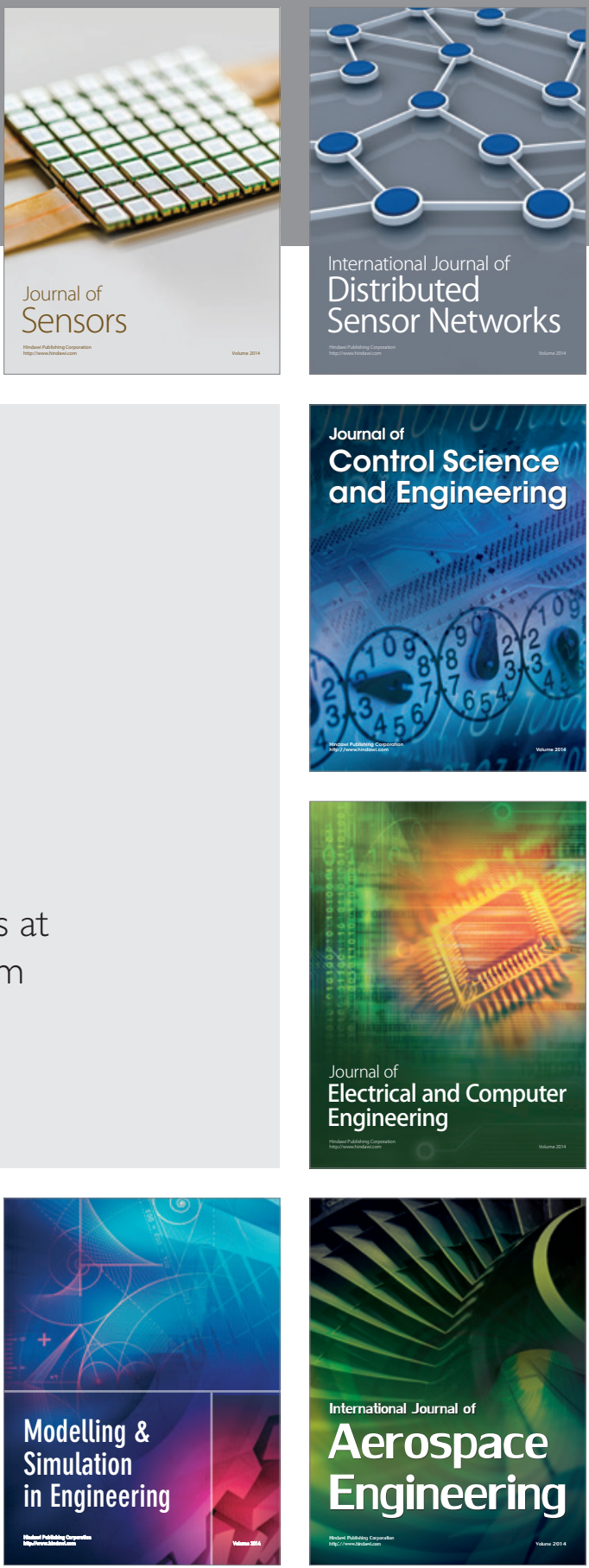

Journal of

Control Science

and Engineering
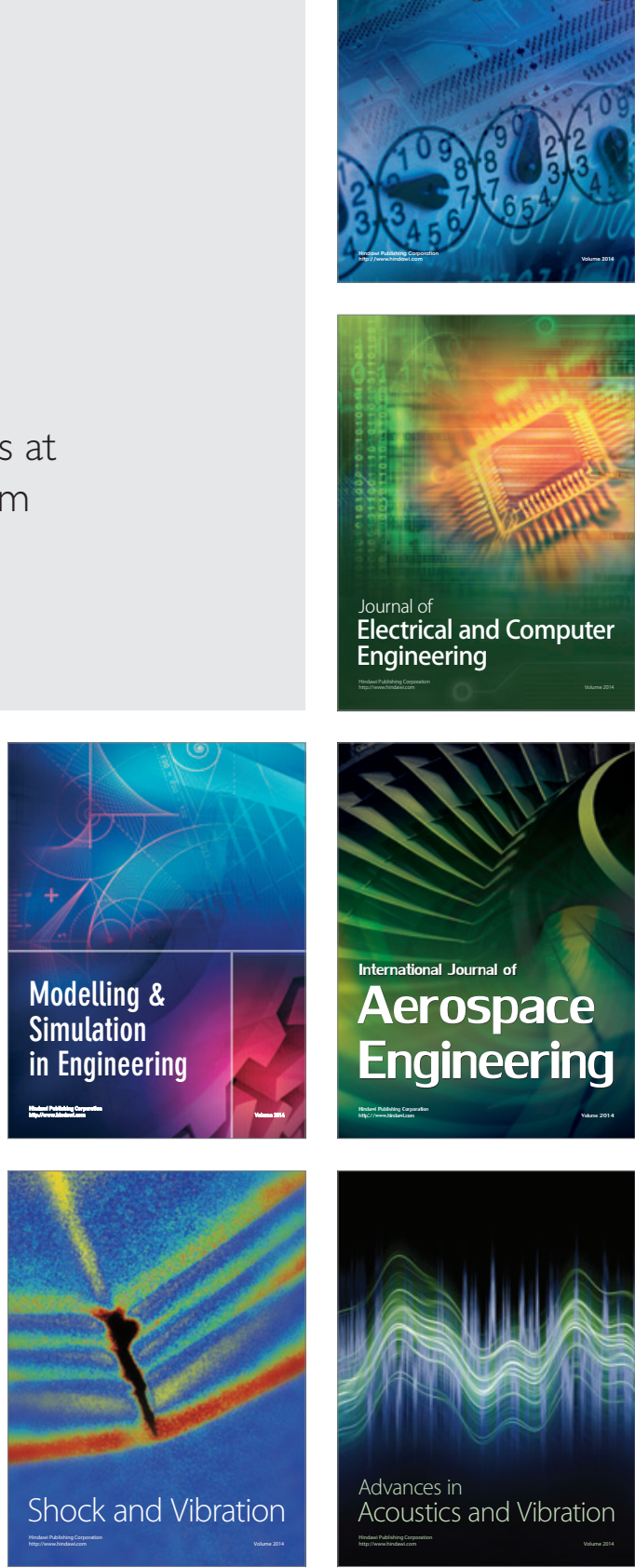